\title{
Sound Wave Diffraction at the Edge of a Sound Barrier
}

\author{
J. PIECHOWICZ
}

Department of Mechanics and Vibroacoustics, AGH — University of Science and Technology

\author{
al. A. Mickiewicza 30, 30-059 Kraków, Poland
}

The diffraction phenomenon is described by the Huygens-Fresnel principle. The review of physical laws ruling the bending of sound waves at the edge of the screen allows the effective selection of both acoustical and geometrical parameters of the screen. Sound wave diffraction theories have been developed on the basis of wave optics, when wavelength is small in comparison to the size of the obstacles, which can be also used in acoustics with the same assumptions about geometry of the system. Diffraction can be seen as a result of the interference of waves reaching the point of observation in accordance with the laws of geometrical optics and wave disturbances arising as a result of interaction with the edge of an obstacle. The paper describes a test method using maximum length sequences for determining the intrinsic characteristics of sound diffraction in situ during testing of roadside noise barriers. A scale model experiment has been performed in an anechoic room. Also, a real noise reducing device was tested in free field conditions.

PACS: 43.20.El, 43.20.Fn, 43.50.Gf, 43.50.Yw, 43.60.Ek, 43.55.Rg

\section{Introduction}

The sound source emits a sound wave that travels toward the acoustic barrier being tested and then it is partly reflected, partly transmitted and partly diffracted by the barrier. Diffraction is the capacity of sound waves to bend at the edge of a barrier. Acoustic wave, when encountering the edge of an obstacle located in its path, is subject to diffraction (bending, deflection). This phenomenon is particularly important, when the noise barriers are used for creation of an acoustic shadow area behind the barrier. The effect of acoustic shadow is practically applied in outdoor locations to reduce the noise from roads or railways. A theoretical basis for description of acoustic wave diffraction and the phenomena taking place in the acoustic shadow area can be found in lectures on three main theories: the Huygens-Fresnel diffraction, the Helmholtz-Kirchhoff diffraction and the geometrical theory of diffraction (GDT). The theories have been developed on the basis of wave optics, i.e. for wavelength small in comparison with the size of obstacles, but they can be also applied in acoustics under the same assumptions regarding the system geometry. According to Huygens principle every point reached by the acoustic wave front becomes a source of a new acoustic wave and the envelope of these new waves is used for construction of the new wave front. Fresnel has developed further the Huygens theory by introducing the effect of interference of acoustic waves being diffracted. In some sense the diffraction phenomenon can be recognized as the interference effect calculated for all the waves reaching the reception point according to the law of geometrical optics combined with additional disturbance (disturbing wave) generated by the interaction with the edge of the obstacle. The mathematical formalism for the diffraction effects has been provided by the Kirchhoff theory, formulated as an element of scalar theory of light. The formula for the Kirchhoff surface integral, fulfilling the Helmholtz equation, can be found using various numerical methods, e.g. boundary element method (BEM)/finite element method (FEM).

The problems of diffraction have been also studied by Prof. Rubinowicz, who in his summary of scientific activity has written the following phrase [1]: "It can be easily proved using the Huygens principle that after separating the geometrical optics radiation from the Kirchhoff wave motion, one obtains for the remaining wave motions an integral running over the shadow border. The outline forms the envelope of a truncated cone. Therefore it was sufficient to integrate over the generating lines (elements) of the cone envelope, which was however hindered by some technical difficulties, to arrive at a formula for the diffracting waves in the closed form. Using that transformation it was also possible to prove a theorem stating that the Kirchhoff diffraction integral solves a uniquely specified problem, which has been described as a step-function problem."

This paper describes a test method for determining the intrinsic characteristics of sound diffraction of added devices installed on the top of traffic noise reducing devices. The test method prescribes measurements of the sound pressure level at several points near the top edge of a noise reducing device with and without the added device installed on its top. The effectiveness of the added device is calculated as the difference between the measured values with and without the added devices. Results are expressed as a function of frequency. A single-number rating is calculated from frequency data. 


\section{Diffraction of an acoustic wave at the edge of the barrier}

One of the problems considered was the acoustic wave diffraction at the barrier's edge. In the diffraction theory published in 1917 Rubinowicz [2] separated the acoustic potential value behind the barrier into two components: the geometric wave potential $\Phi^{\mathrm{g}}(P)$ and the diffracting wave potential $\Phi^{\mathrm{d}}(P)$. Such a solution was then analytically extended to the area before the barrier by specifying [1]:

$$
\Phi(P)=\Phi^{\mathrm{g}}(P)+\Phi^{\mathrm{d}}(P),
$$

where $\Phi^{\mathrm{g}}(P)=\Phi_{0}(P)$ - for the area reached by the direct wave, $\Phi^{\mathrm{g}}(P)=0$ - for the shadow area.

For a semi-infinite barrier, in the form of a half-plane, the formulae describing the diffracting wave can be found in [1]. The Rubinowicz method allows the construction of a solution that is valid in the whole space with exclusion of the plane in which the barrier is located, for the high frequency range. It also allows taking into account the reflected waves.

In the sixties of the previous century, Keller [3] published the foundations of the geometrical theory of diffraction. It was based on the previous works by Young, who was the first to propose that the diffraction problem should be treated as an interference problem of the wave produced at the barrier's edge and the wave for which the propagation path can be determined by geometry [4]. The GDT enhances the optical geometry problems by adding the diffraction phenomena. Starting from the general Fermat principle Keller was able to indicate the possibility of acoustic field prediction in the acoustic shadow zone of the barrier by a simplified treatment, namely by summation of all acoustic rays reaching the specific point. The method treats the barrier's edge as a second source of the acoustic rays, which can radiate into the acoustic shadow zone, and these rays can be consecutively reflected or diffracted before they reach the observer's ear. The GDT method can be successfully applied to diffraction problems on a single edge, but also to problems of diffraction on the corners of buildings or the edges of noise barriers.

The diffraction is a form of sound wave scattering by the objects, the size of which is comparable to the wavelength of the sound wave. Therefore it is a fundamental aspect of the sound wave propagation, as the wavelength of the sound waves lies in the range between $0.2 \mathrm{~m}$ and $17 \mathrm{~m}$. Without including the diffraction into the modeling of acoustic phenomena around the noise barriers the simulation results are not consistent with the experimental results. Employing the assumptions of GDT one can confront the calculated results with the values obtained from the experiment for selected reception points behind the barrier.

In practical realizations the barriers used in the open space are attributed the functions of noise barriers. Learning the consequences of the acoustic wave diffraction on the barrier's edge allows an effective selection of parameters (both acoustic and geometric) of the barrier. Using an appropriate measurement technique one can determine the diffraction components of the acoustic signal transferred to the acoustic shadow zone. In particular one can do it using the impulse response for a given "sound source-barrier-microphone" measurement system.

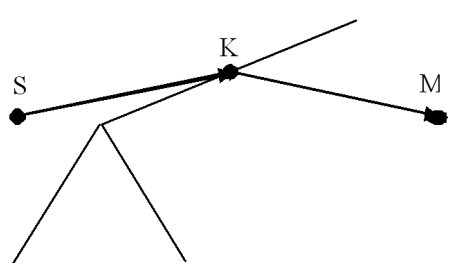

Fig. 1. Layout for the diffraction effects on the barrier's edge. $S$ is the sound source, $M$ is the reception point, $K$ is the diffraction point at the barrier's edge.

Sound transfer to the zone behind the noise barrier is directly related to the diffraction of the acoustic wave. For the harmonic wave the sound pressure in the field of the sound wave diffracted on the barrier's edge can be presented in relation to incident wave field (Fig. 1) in the following form [5]:

$$
p_{\text {diff }}(M)=p_{\text {inc }}(K) D_{\mathrm{I}} A\left(r_{\mathrm{S}}, r_{\mathrm{R}}\right) \mathrm{e}^{-\mathrm{i} k r},
$$

where $M$ is the reception point, $K$ is the diffraction point at the barrier's edge, $k$ is the wave number $k=2 \pi / \lambda$, $A\left(r_{\mathrm{S}}, r_{\mathrm{R}}\right)$ is the attenuation along the propagation path of the acoustic wave, $D_{\mathrm{I}}$ is the diffraction index calculated from the GDT.

Determination of the diffraction index allows a quantitative evaluation of the sound field behind the noise barrier.

\section{Measurement procedure}

As a part of the European Research Program "Adrienne" new methods have been defined for determination of intrinsic characteristics of road traffic noise reduction barriers in situ [6]. The methods are based on analysis of the impulse response of the measurement systems, in which the main component is the construction of the noise reduction barrier. The determined characteristics are: reflection coefficients, acoustic insulating power for the barrier panels and the sound diffraction index. In the specific technical tasks a very important problem is the evaluation of the effects of the barrier's top cover on its acoustic insulating properties. The barriers are mainly constructed in the form of plain walls, however in some cases special elements (added devices) are mounted on its top edge, which are intended to improve the acoustics characteristics of the barrier. For evaluation of the actual effect of the added devices on the sound propagation behind the wall, special methods have been elaborated for measurement of the diffraction index [7]. The value of the diffraction index has been defined as a ratio of the 
pressure of the diffracted acoustic wave to the pressure of the direct acoustics wave. The diffraction index $D I$ in octave frequency bands is determined according to the following formula:

$D I=-10 \log \left[\frac{\sum_{i=1}^{n} \int_{\Delta f i}\left|F\left[h_{d k}(t) w_{d k}(t)\right]\right|^{2} \mathrm{~d} f\left(\frac{d_{k}}{d_{i}}\right)^{2}}{n \sum_{i=1}^{n} \int_{\Delta f i}\left|F\left[h_{i}(t) w_{i}(t)\right]\right|^{2} \mathrm{~d} f}\right]$,

where $h_{i}(t)$ is the reference free-field component; $h_{d k}(t)$ is the component of the impulse response diffracted by the top of edge of the test construction and received at the $k$-th impulse response; $d_{i}$ is the geometrical spreading correction factor for the reference free-field component; $d_{k}$ is the geometrical spreading correction factor for the diffracted component and received at the $k$-th measurement point $(k=1, \ldots, n) ; w_{i}(t)$ is the incident reference free field component time window; $w_{t, k}(t)$ is the time window for the diffracted component at the $k$-th measurement point; $F$ is the symbol of the Fourier transform; $\Delta f_{j}$ is the $j$-th one-third octave frequency band (from $100 \mathrm{~Hz}$ to $5 \mathrm{kHz}$ ); and $n=8, \ldots, 36$ is the number of measurement points.

The scheme of the measurement setup is presented in Fig. 2. Pseudo-random maximum length sequences (MLS) signal was generated in the system consisting of a generator, amplifier and a loudspeaker.

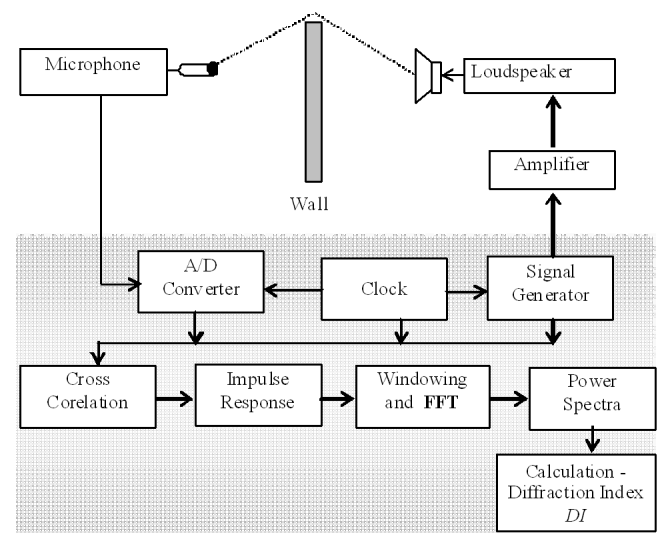

Fig. 2. Scheme of the measurement setup for determination of the diffraction index.

The impulse response has been registered by a microphone located in the measurement points behind the acoustic barrier, and then the signal was processed in both time and frequency domains. The geometry of the measurement points layout and the relative locations of the sound source and the microphone are shown in Fig. 3. The measurements have been carried out for 36 various locations of the sound source and the measurement microphone in relation to the top edge of the barrier and various locations of the reception points behind the barrier. The measurement procedure has been repeated six times for every loudspeaker location with perpendicular orientation of the measuring system (see Fig. 3a), and then measurement system was turned by $45^{\circ}$ and the whole measurement procedure was repeated (see Fig. 3b).

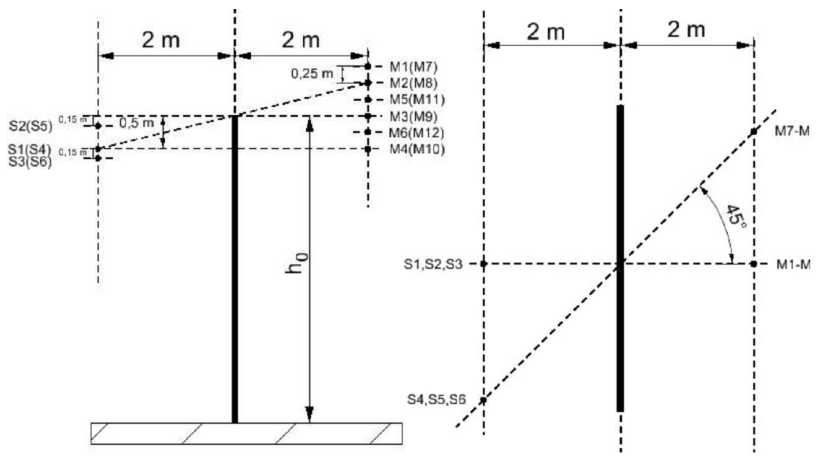

Fig. 3. Locations of the sound source and the noise reception points [7].

In one series of measurements the impulse response functions were determined for reference measurements in free-field for two locations of the loudspeaker-microphone system.

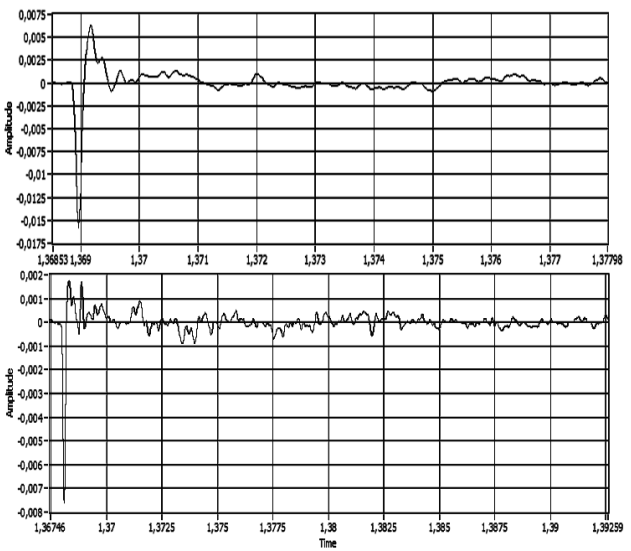

Fig. 4. Impulse response function for measurement setup with a given reception point.

Figure 4 presents the impulse response function for the reference measurement (a) and a specific layout of $S 3-M 6$ points. The analysis of the impulse response in all 36 measurement points has been carried out in $1 / 3$ octave frequency bands. The complete measurement procedure has been realized in software in the LabView environment. The experiments in the laboratory conditions have been carried out using a wooden model of the acoustic barrier, of the $1.8 \mathrm{~m}$ height, in free-field conditions in anechoic chamber. The barrier is located near the center of the room, the lateral sound propagation paths were secured using by sound-insulation plates. Figure 5 shows the top edge of the examined barrier.

\section{Results for the diffraction index}

The experimental studies were carried out for two types of barrier surface coatings, applied from sound 


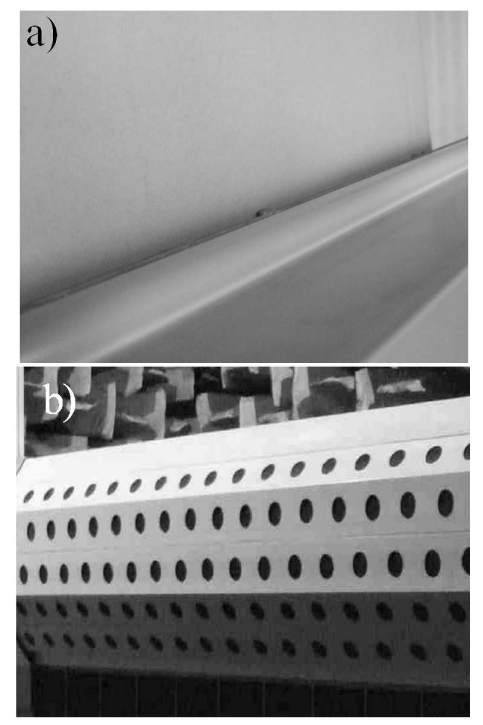

Fig. 5. The examined object: (a) top edge of the barrier, (b) octagon - an added device.

source side. In one case the barrier wall has been coated with a reflecting layer, while in the other case it was an absorbing layer - the wall has been covered with polyurethane foam tiles. The plots present the diffraction indices for $1 / 3$ octave frequency bands for six various locations of the sound source and two directions of the wave propagation (Figs. 6, 7). The index value for each sound source location was averaged over the 6 locations of the reception points referring to the microphone positions $M 1-M 6$ and $M 7-M 12$, respectively. The sound source locations $S 1, S 2, S 3$ are used for direction perpendicular to the barrier surface, while the sound locations $S 4, S 5$, $S 6$ are used for direction askew $\left(45^{\circ}\right.$ angle) to the barrier wall (Fig. 3). The value of the diffraction index depends on the elevation of the sound source location with respect to the top edge of the barrier. The smallest values of the index have been obtained for the sound source localizations $S 2$ and $S 5$ (15 cm below the top edge of the barrier). The plots of all the curves are similar irrespective of the barrier wall coating (reflecting or absorbing layer). The characteristics of the diffraction index for both types of the barrier wall coating are presented in Fig. 8 (averaged value of the diffraction index for all 36 measurement points - according to formula (3)). The plots of the indices in $1 / 3$ octave frequency bands do not differ much between these two cases. In the middle frequency range, i.e. $800-3150 \mathrm{~Hz}$, the index values are slightly greater for the reflective barrier coating.

Figures 9 and 10 present the plots of diffraction index for two far out microphone locations; the $M 1$ position $75 \mathrm{~cm}$ above the top edge of the barrier and position $M 4$ - $50 \mathrm{~cm}$ below the top edge. In the measurement point $M 1$ the sound pressure is first of all a sum of the two pressures: from the direct wave and the wave diffracted by the edge of the barrier, while for the microphone loca-

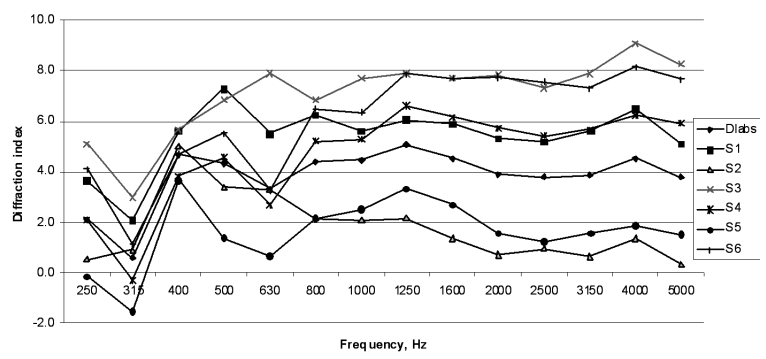

Fig. 6. Diffraction indices for $1 / 3$ octave frequency bands, for the absorbing barrier coating, for various sound source locations.

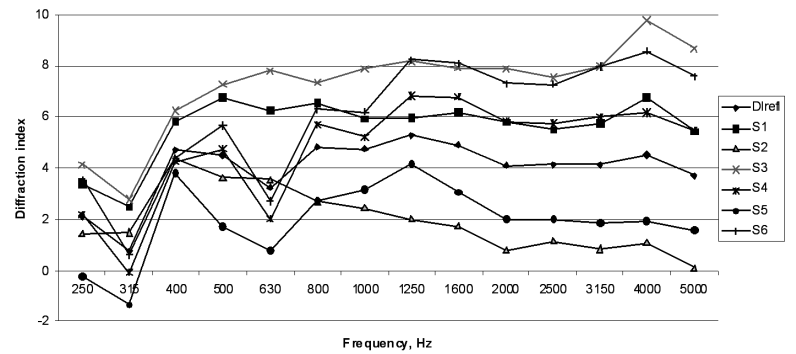

Fig. 7. Diffraction indices for $1 / 3$ octave frequency bands, for the reflective barrier coating, for various sound source locations.

tion $M 4$ it is mainly the pressure of the wave diffracted by the barrier's edge. Therefore the diffraction index value in point $M 4$ is much bigger than for reception point $M 1$.

In the present study of the diffraction effects measurements have been carried out with special added devices mounted on the top edge of the barrier. Modification of the acoustic characteristics of the barrier comprises mainly the change in diffraction conditions at the top edge of the barrier. The additional construction called octagon (see Fig. 5), with perforated wall surface on the sound source side and filled inside with a mineral wool layer, has been mounted on the top edge of the barrier. For comparison of the device's effect on propagation of the acoustic wave behind the wall diffraction index $D I_{0}$ has been measured without the added device and then

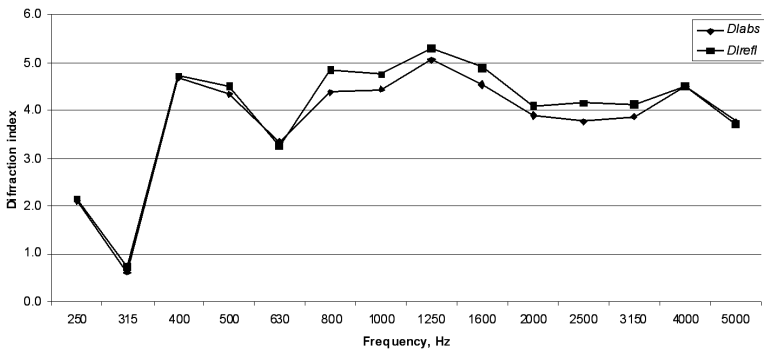

Fig. 8. Averaged value of the diffraction index for 36 measurement points for both reflective and absorbing barrier coatings. 


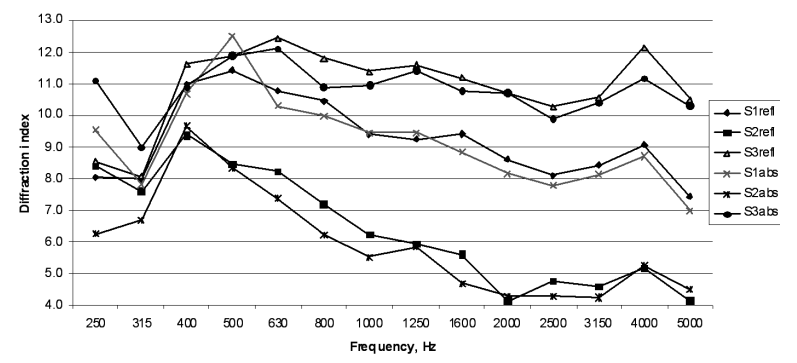

Fig. 9. Characteristics of the diffraction index in $1 / 3$ octave frequency bands for point $M 1(0.75 \mathrm{~m}$ above the top edge of the barrier) and 3 various locations of the sound source $S 1, S 2, S 3$ (wall surface both reflecting and absorbing).

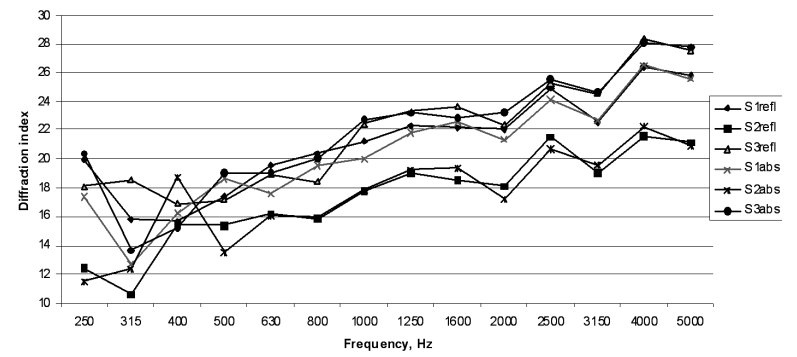

Fig. 10. Characteristics of the diffraction index in $1 / 3$ octave frequency bands for point $M 4(0.5 \mathrm{~m}$ below the top edge of the barrier) and 3 various locations of the sound source $S 1, S 2, S 3$ (wall surface both absorbing and reflecting).

measured again, as $D I_{\text {ad }}$, with the added device mounted at the barrier's edge. Diffraction index difference $\Delta D I$ is a difference between the results of sound diffraction test on the same reference wall with $\left(D I_{\mathrm{ad}}\right)$ and without $\left(D I_{0}\right)$ an added device on the top described by formula [7]:

$$
\Delta D I=D I_{\mathrm{ad}}-D I_{0} .
$$

A single-number rating shall be derived to indicate the performance of the product. The individual diffraction index values shall be weighted according to the normalized traffic noise spectrum defined in EN 1793-3.

A single-number diffraction difference index is zero for both types of the acoustic barrier walls (Fig. 11), which could indicate a neutral behavior of the octagon is formation of the diffraction sound field behind the acoustic barrier.

\section{Conclusions}

The sound diffraction effect considerably depends on the ratio of the sound wavelength to the characteristic size of the obstacle. The application of the pseudo-random MLS signal and the analysis employing the impulse response function allowed completing of the acoustic studies of the barrier for a model system located in

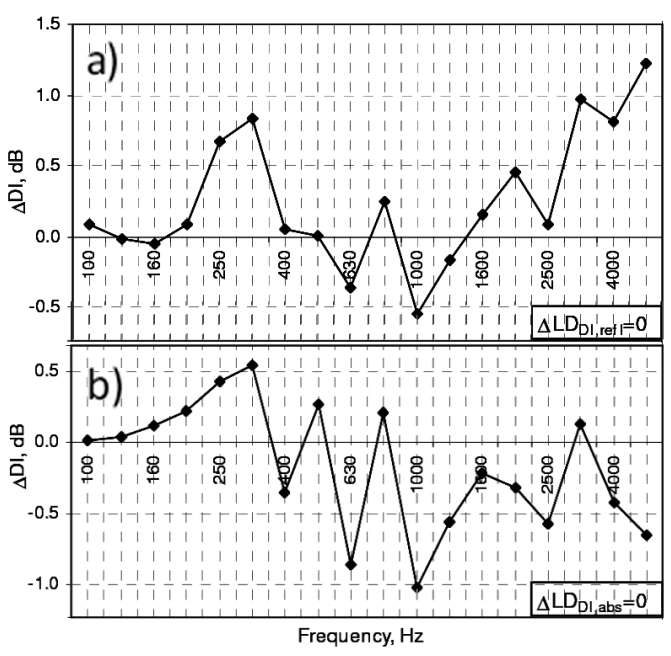

Fig. 11. Plots of diffraction index differences are presented for reflecting (a) and absorbing (b) barrier walls.

the anechoic chamber as well as for in situ conditions, in the place where the barrier will be installed. The presented results of the study show the effects of acoustic wave diffraction on the barrier's edge on the formation of the sound field behind the barrier. The effect of acoustic shadow degradation behind the barrier is of great importance for the effectiveness of the noise barrier application for environment noise protection purposes. The study indicates that the barrier surface coating at the sound source side (reflecting, absorbing layer) does not considerably affect the sound wave diffraction, when the other conditions at the top edge of the barrier are kept identical. The studies of the octagon shaped added device presented in the literature [6] have been confirmed by the experimental results, where a slight effect has been found on the sound pressure values measured behind the barrier in relation to the results for a plain barrier without added devices. In order to make the effects of added devices clearly noticeable in the practical barrier designs complex geometrical structures are used like e.g. reindeer type [8]. The results of the laboratory tests have been compared with the real acoustic barrier of the same type, $4 \mathrm{~m}$ in height and $100 \mathrm{~m}$ in length in its actual location, on which the octagon structure has been mounted on half of its length. In both experiments, in laboratory and in situ significant consistence has been achieved for the final results of the diffraction index.

\section{Acknowledgments}

This research work is supported by the Polish Ministry of Science and Higher Education grant no. N N 504342536 .

\section{References}

[1] W. Rubinowicz, Adv. Phys. 21, 161 (1970) (in Polish). 
[2] Z. Engel, J. Sadowski, M. Stawicka Wałkowska, S. Zaremba, Noise Barriers, AGH, Kraków 1990 (in Polish).

[3] J.B. Keller, J. Opt. Soc. Am. 62, 116 (1962).

[4] P.B. Sunil Kumar, G.S. Ranganath, Current Sci. 61, 10 (1991).

[5] D.A. McNamara, C.W.I. Pistorius, J.A.G. Malherbe, Introduction to the Uniform Geometrical Theory of Diffraction, Artech House, Norwood 1990.

[6] M. Garai, P. Guidrzi, in: Proc. 19th Int. Congress on Acoustics, Madrid, 2007, CD.
[7] CEN/TS 1793-4 Road traffic noise reducing devices - Test method for determining the acoustic performance - Part 4: Intrinsic characteristics - in situ values of sound diffraction.

[8] H. Tachibana, in: Proc. 39th Int. Congress on Noise Control Engineering, Lisbon, 2010, CD. 